\title{
ALK Rearrangement in Small-Cell Lung Cancer and Durable Response to Alectinib: A Case Report
}

\author{
Ning Sun ${ }^{1, *}$ \\ Yan Zhuang ${ }^{1} *$ \\ Junling Zhang $\mathbb{D}^{2}$ \\ Shiqing Chen $\mathbb{D}^{2}$ \\ Yuwen $\mathrm{Dai}^{2}$ \\ Renhong Guo' \\ 'Department of Medical Oncology, \\ Jiangsu Cancer Hospital, Jiangsu Institute \\ of Cancer Research, The Affiliated \\ Cancer Hospital of Nanjing Medical \\ University, Nanjing, People's Republic of \\ China; ${ }^{2}$ The Medical Department, 3D \\ Medicines Inc., Shanghai, People's \\ Republic of China \\ *These authors contributed equally to \\ this work
}

Background: With the development of next-generation sequencing (NGS), several anaplastic lymphoma kinase $(A L K)$ fusion partner genes have been identified. However, $A L K$ fusion is extremely rare in small cell lung cancer (SCLC), and there is no standard treatment option. Here, we report a patient with SCLC who carried an $A L K$ - Intergenic Region (IR) rearrangement and responded to Alectinib.

Case Presentation: A 26-year-old man was pathologically diagnosed with extensive-stage SCLC. After 2 cycles of first-line chemotherapy, CT showed a large soft tissue mass in the middle lobe of the right lung and increased liver nodules, left kidney lesions and right kidney lesions. To seek potential therapeutic regimens, $A L K$ rearrangement was identified. The patient achieved a rapid and durable partial response with Alectinib (600 mg BID). The patient experienced a significant clinical response with a progression-free survival of more than 6 months. There were no grade 3 or more adverse events reported, and there was no dose reduction during treatment. Following Alectinib treatment, the allele frequency of $A L K$ rearrangement and $R B 1$ and TP53 mutations in plasma circulating tumor DNA decreased with the reduction in tumor size.

Conclusion: This case provides a meaningful reference for the treatment of SCLC patients with $A L K$ rearrangement. This case also provides valuable information on the response to ALK inhibitors in patients with $A L K-I R$ rearrangement and better understanding of ALKTKI applications in the future.

Keywords: small-cell lung cancer, $A L K$ rearrangement, ALK inhibitor, immunohistochemistry, genetics

\section{Introduction}

Lung cancer is the most common cause of cancer-related death, with small cell lung cancer (SCLC) accounting for about $3.7 \%$ of all lung cancers in China. ${ }^{1,2}$ Although SCLC is highly sensitive to chemotherapy and radiotherapy, the median overall survival (OS) for extensive SCLC patients is only 8-13 months. ${ }^{3}$

Over the past decade, oncogenic driver gene mutations have been identified in patients with lung cancer, and these findings have led to a paradigm shift in lung cancer diagnosis and treatment. For example, anaplastic lymphoma kinase $(A L K)$ has been shown to play an important role in tumorigenesis, survival, and proliferation in lung cancer. $A L K$ fusion mutations have attracted attention as potential targets of ALK kinase inhibitors. In the ALEX study, Alectinib compared with Crizotinib in first-line treatment of $A L K$-positive NSCLC showed prolonged median OS and has become the first-line option for $A L K$-positive NSCLC patients. ${ }^{4}$
Correspondence: Renhong Guo Department of Medical Oncology, Jiangsu Cancer Hospital, Jiangsu Institute of

Cancer Research, The Affiliated Cance Hospital of Nanjing Medical University, Nanjing, 210009, People's Republic of China

Email guorh610@sina.com 
With the development of next-generation sequencing (NGS), several $A L K$ fusion partner genes, such as EML6$A L K, T M E D 2-A L K$ and MYTIL-ALK, have been identified. ${ }^{5}$ However, $A L K$ fusion is extremely rare in SCLC, and there is no standard treatment option. To the best of our knowledge, only three rare cases of $A L K$ fusion with SCLC have been previously reported. ${ }^{6-8}$ Hence, we report a patient of SCLC harboring an $A L K$ rearrangement and describe the patient's clinical course and response to Alectinib.

\section{Case Descriptions}

A 26-year-old non-smoking male was referred to our hospital due to chest tightness, wheezing, and irritant dry cough. Chest computed tomography (CT) revealed a large, soft tissue mass of $12.43 \times 10.22 \mathrm{~cm}$ in the right middle lobe and a massive right pleural effusion (Figure 1). Abdominal CT showed liver hypodensity with a nodule measuring 2.83 $\times 2.76 \mathrm{~cm}$ (Figure 1). Local hypodensity shadow of the kidney was present on both sides, with a mass of $4.53 \times$ $4.26 \mathrm{~cm}$ in the left kidney and $4.16 \times 3.36 \mathrm{~cm}$ in the right kidney (Figure 1). A CT-guided tumor biopsy was then performed, and the tumor was pathologically diagnosed as SCLC (extensive-stage, stage IV, Figure 2A) with immunoreactivity to CD56, Synaptophysin (Syn), CgA and Ki-67 (about $90 \%$ positive), and negative for TTF-1 and $\mathrm{p} 40$ on (Figure 2B-G). In addition, laboratory examinations revealed elevations in the levels of neuron-specific enolase (NSE; $179 \mathrm{ng} / \mathrm{mL}$ ), whereas no abnormalities were observed in carbohydrate antigen 19-9 (CA19-9) and carcinoembryonic antigen (CEA). He was treated with chemotherapy (etoposide plus lobaplatin) two cycles. On October 9, 2020, the patient showed a progressive disease with lung lesion (from $12.43 \times 10.22 \mathrm{~cm}$ to $15.00 \times 12.6 \mathrm{~cm}$ ), liver nodule (from $2.83 \times 2.76 \mathrm{~cm}$ to $4.16 \times 3.36 \mathrm{~cm}$ ), left kidney lesion (from $4.53 \times 4.26 \mathrm{~cm}$ to $8.06 \times 6.8 \mathrm{~cm}$ ), and right kidney lesion (from $4.16 \times 3.36 \mathrm{~cm}$ to $6.26 \times 4.52 \mathrm{~cm}$ ). Meanwhile, the levels of NSE ( $>300 \mathrm{ng} / \mathrm{mL})$, CA19-9 $(24.8 \mathrm{U} / \mathrm{mL})$ and CEA $(2.13 \mathrm{ng} / \mathrm{mL})$ were increased (Figure $2 \mathrm{H}$ and I).

To seek potential therapeutic regimens, the blood specimen was subjected to NGS analysis, and a novel $A L K$ and intergenic region (IR) rearrangement was identified (mutant allele frequency [MAF]: 19.21\%, Figure $3 \mathrm{~A}$ and $\mathrm{B}$ ). Immunohistochemical staining (Ventana Medical Systems, Tucson, AZ) showed an increased signal of ALK expression (Figure 3C). Alectinib (600 mg BID) combined with irinotecan (CPT-11; 0.4g dl) as second-line therapy was administered. After two cycles of treatment, CT scan showed a partial response $(\mathrm{PR})$ with a reduction from $15.0 \times 12.6 \mathrm{~cm}$ to $8.42 \times$

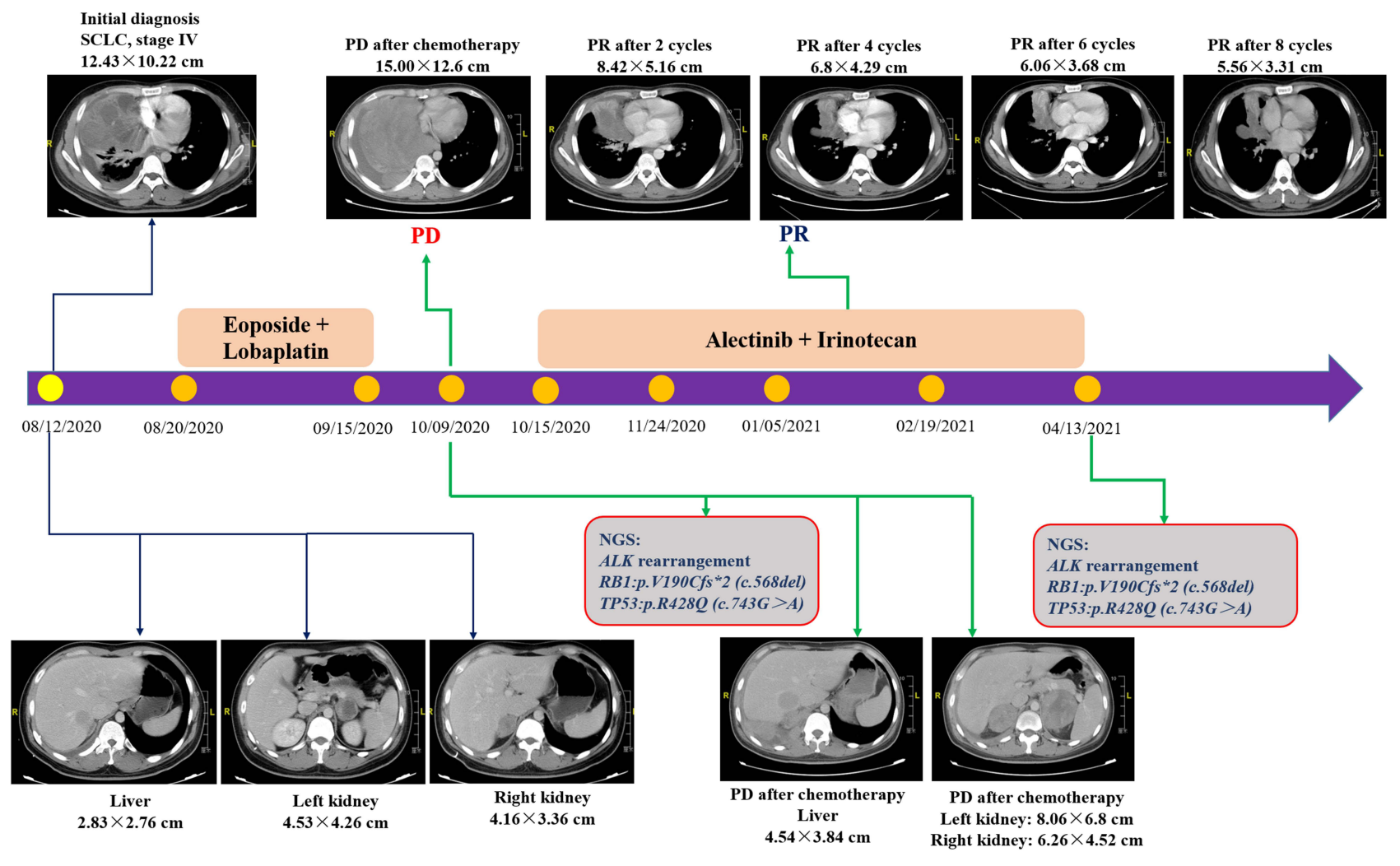

Figure I Timeline of the clinical course in this patient. 

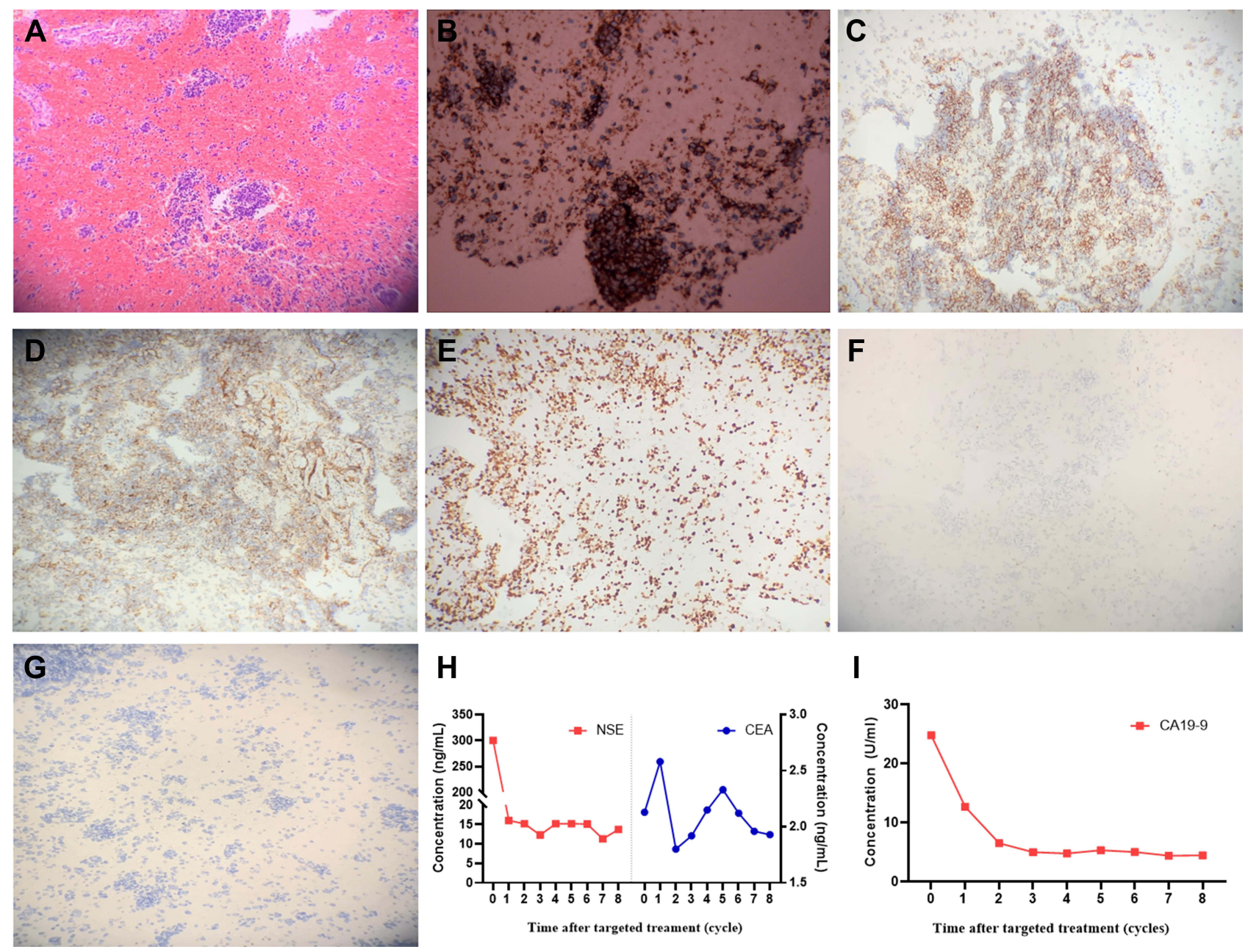

Figure 2 Multi-biomarker immunohistochemistry confirms pathological typing of small cell lung cancer. (A) HE, (B) CD56, (C) Syn, (D) CgA, (E) Ki-67, (F) TTF-I, (G) p40, (H) CEA and NSE, (I) CAI9-9.

$5.16 \mathrm{~cm}$ for lung lesion (Figure 1). Meanwhile, the size of liver tumor $(2.0 \times 1.57 \mathrm{~cm})$ and bilateral kidney tumor (left: $3.3 \times 2.37 \mathrm{~cm}$; right: $3.05 \times 2.18 \mathrm{~cm}$ ) were decreased (Figure 4). NGS-based ctDNA test showed a decrease in MAF of $A L K$ rearrangement (from $19.21 \%$ to $0.58 \%$, Figure 3B). During the 4th, 6th and 8th cycles after treatment, the tumor lesions continued to decrease, and the patient maintained PR (Figures 1 and 4). In addition, $A L K$ rearrangement had disappeared after 4 cycles of treatment (Figure 3B). There were no obvious adverse reactions to the treatment. It suggests that the patient has a durable response to Alectinib and achieved 6-month progression-free survival (PFS) until the report date of this case.

\section{Discussion}

This is the first reported SCLC patient with $A L K$ rearrangement who benefited from the treatment of Alectinib plus CPT-11. This fusion contains $A L K$ kinase domain, which might drive oncogenesis. In our case, strong ALK protein expression is further observed with this patient. This patient achieved PR after 2 cycles of Alectinib plus CPT11 and had a durable response to ALK inhibitors and achieved 6-month PFS untill the report date of this case.

Although SCLC is a class of aggressive tumors that are clinically sensitive to chemotherapy, the median PFS for first-line treatment was 4.7-6.9 months. ${ }^{9,10}$ However, this patient developed PD after receiving 2 cycles of etoposide plus lobaplatin chemotherapy, and the PFS was significantly lower than that of the historical control group. As a reason for the poor clinical course of current patients, the fusion gene may affect the sensitivity of first-line chemotherapy. In addition, it has been reported that platinum/etoposide chemotherapy-resistant SCLC has an extremely low chance of responding to second-line chemotherapy with poor OS. ${ }^{11}$ This is one of the reasons why we chose CPT-11 combined with Alectinib for this $A L K$ rearrangement SCLC. 
A

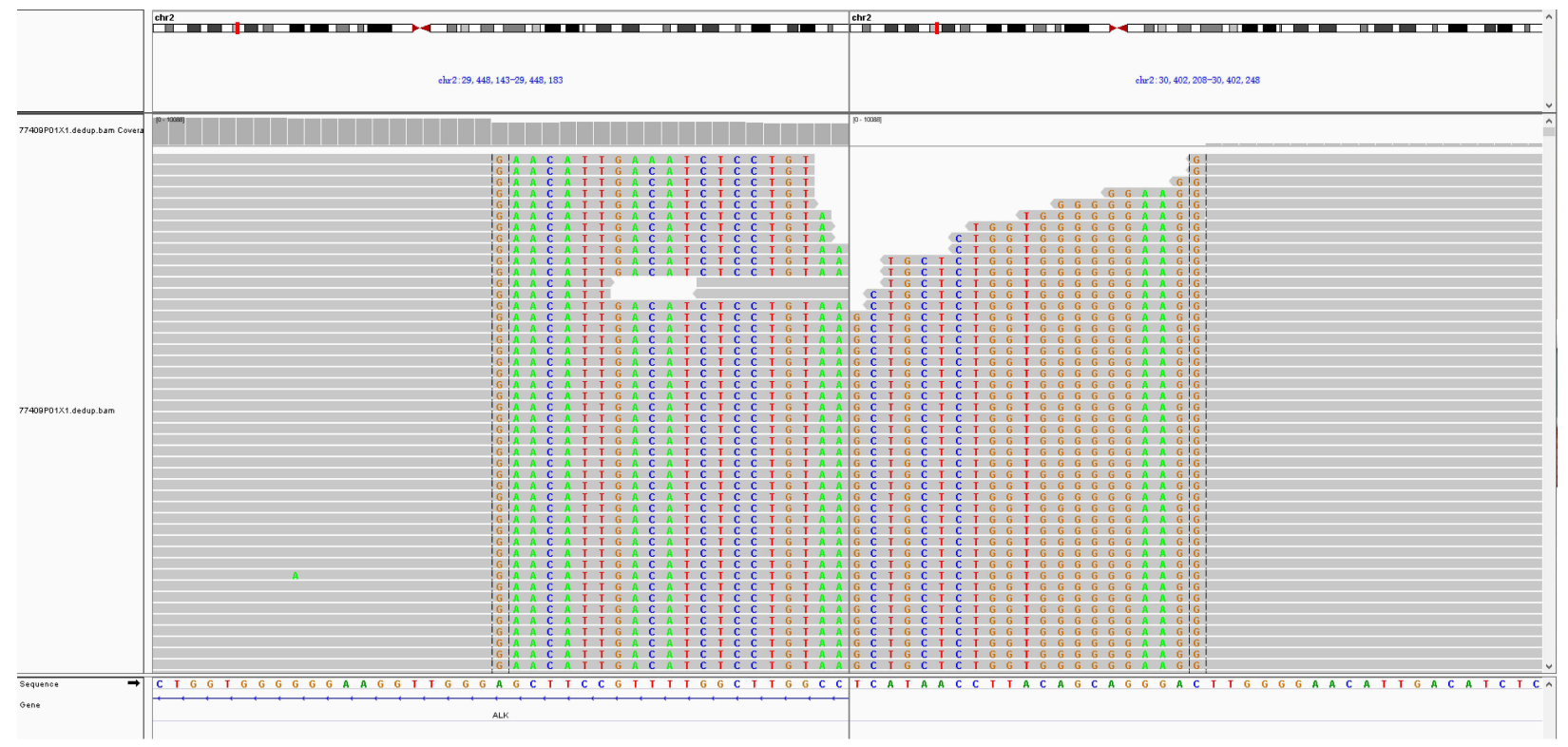

B

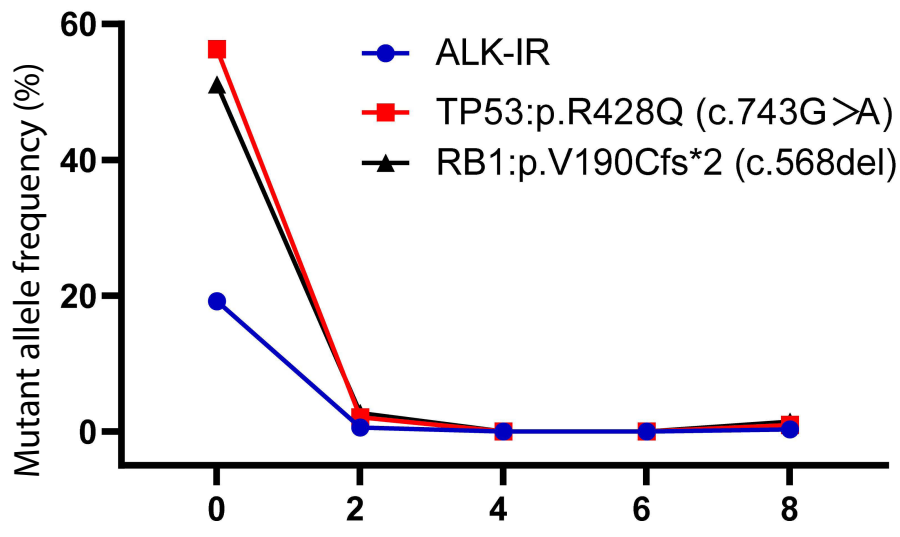

Time after targeted treatment (cycle)
C

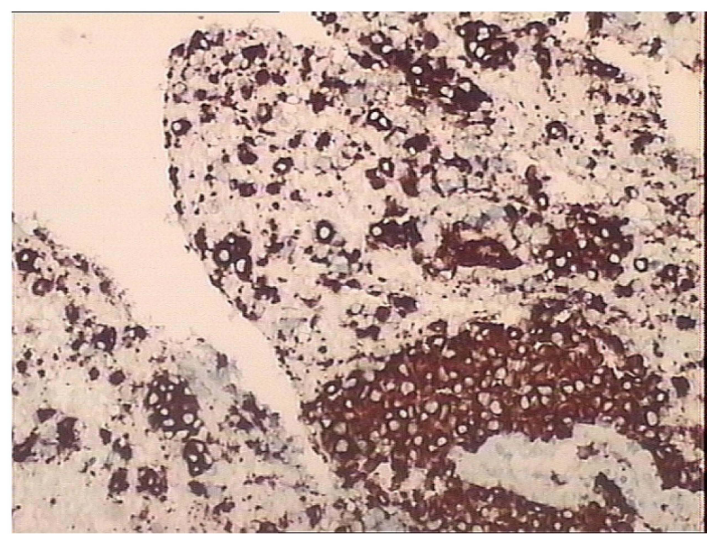

Figure 3 Next-generation sequencing results. (A) Next-generation sequencing analysis of ALK fusion mutation in the patient's biopsy sample. (B) The MAF of mutated genes. (C) Immunohistochemical staining of ALK expression.

One of the limitations of the current case report is that the $A L K$ partner gene is not clear. Although NGS-based methodology has a high specificity and is likely to detect fusions with novel partners, RNA-based NGS is preferable to DNAbased NGS for fusion detection according to the NCCN Guidelines Version 3.2021 for non-small cell lung cancer. However, for this patient, there are insufficient tissue samples to perform RNA-based NGS testing. In order to find a better treatment, the patient used blood samples for DNA-based NGS testing and verified ALK positive with IHC methodology. In addition, CPT-11 plus Alectinib is not the standard of care for second-line treatment of $A L K$-positive SCLC. Formally, we cannot rule out the dependence of disease response on CPT-11 administration. Therefore, whether CPT-11 is maintained in all Alectinib treatments needs to be further confirmed in prospective studies.

In this article, we reported for the first time a case of SCLC failed chemotherapy with $A L K$ rearrangement and benefited from treatment with Alectinib. Although the frequency and significance of the ALK rearrangement in SCLC patients have not been determined, this phenomenon suggests that SCLC patients harboring the $A L K$ rearrangement can be successfully 


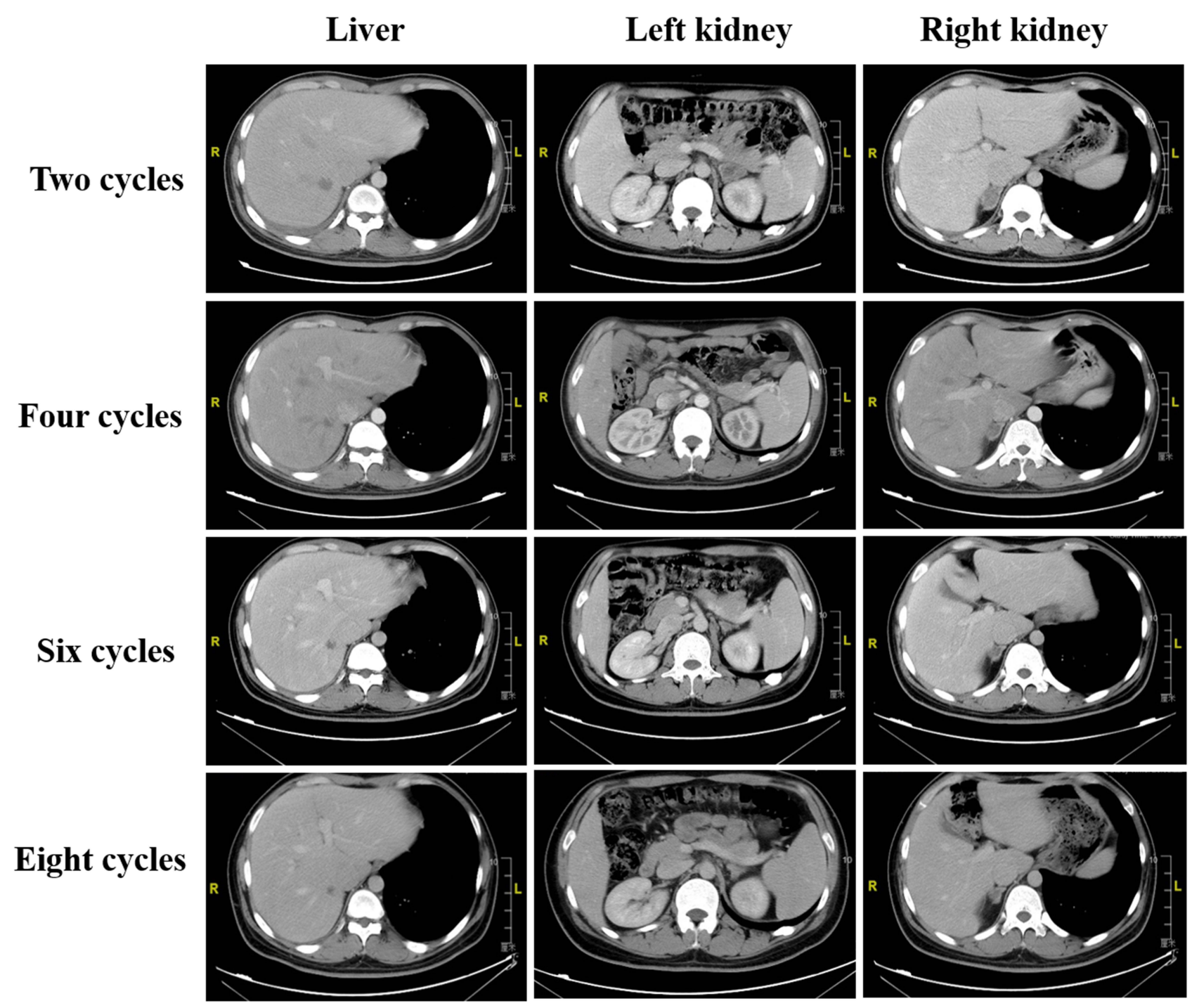

Figure 4 Computed tomography scan of metastatic lesions after treatment.

treated with ALK inhibitors. This case has provided valuable insights into the future cancer treatment in clinic.

\section{Data Sharing Statement}

All data generated or analyzed during this study are included in this published article.

\section{Ethics Approval and Consent to Participate}

This study was approved by the Ethics Committee of Jiangsu Cancer Hospital.

\section{Consent for Publication}

The patient provided written informed consent for the case details to be published.

\section{Funding}

There is no funding to report.

\section{Disclosure}

Ning Sun and Yan Zhuang are co-first authors for this study. Junling Zhang, Shiqing Chen, Yuwen Dai are employees of 3D Medicines Inc. The authors declare no other conflicts of interest in this work.

\section{References}

1. Bray F, Ferlay J, Soerjomataram I, et al. Global cancer statistics 2018: GLOBOCAN estimates of incidence and mortality worldwide for 36 cancers in 185 countries. CA Cancer J Clin. 2018;68:394-424. doi:10.3322/caac. 21492

2. Tian HX, Zhang XC, Yang JJ, et al. Clinical characteristics and sequence complexity of anaplastic lymphoma kinase gene fusions in Chinese lung cancer patients. Lung Cancer. 2017;114:90-95. doi:10.1016/j.lungcan.2017.11.001 
3. Puglisi M, Dolly S, Faria A, et al. Treatment options for small cell lung cancer - do we have more choice? $\mathrm{Br} J$ Cancer. 2010;102:629-638. doi:10.1038/sj.bjc.6605527

4. Mok T, Camidge DR, Gadgeel SM, et al. Updated overall survival and final progression-free survival data for patients with treatment-naive advanced ALK-positive non-small-cell lung cancer in the ALEX study. Ann Oncol. 2020;31:1056-1064. doi:10.1016/j. annonc.2020.04.478

5. Mao W, Chen R, Zhang J, et al. TMED2-ALK, a novel ALK fusion gene identified in a patient with lung adenocarcinoma. $J$ Thorac Oncol. 2020;15:e37-e39. doi:10.1016/j.jtho.2019.10.020

6. Toyokawa G, Takenoyama M, Taguchi K, et al. An extremely rare case of small-cell lung cancer harboring variant 2 of the EML4-ALK fusion gene. Lung Cancer. 2013;81:487-490. doi:10.1016/j.lungcan.2013.05.022

7. Li T, Zhang F, Wu Z, et al. PLEKHM2-ALK: a novel fusion in small-cell lung cancer and durable response to ALK inhibitors. Lung Cancer. 2020;139:146-150. doi:10.1016/j.lungcan.2019.11.002
8. Toyokawa G, Taguchi K, Ohba T, et al. First case of combined small-cell lung cancer with adenocarcinoma harboring EML4-ALK fusion and an exon 19 EGFR mutation in each histological component. $J$ Thorac Oncol. 2012;7:e39-e41. doi:10.1097/ JTO.0b013e3182762bcb

9. Noda K, Nishiwaki Y, Kawahara M, et al. Irinotecan plus cisplatin compared with etoposide plus cisplatin for extensive small-cell lung cancer. $N$ Engl J Med. 2002;346:85-91. doi:10.1056/NEJMoa003034

10. Okamoto H, Watanabe K, Kunikane H, et al. Randomised Phase III trial of carboplatin plus etoposide vs split doses of cisplatin plus etoposide in elderly or poor-risk patients with extensive disease small-cell lung cancer: JCOG 9702. Br J Cancer. 2007;97:162-169. doi:10.1038/sj.bjc.6603810

11. Ardizzoni A, Tiseo M, Boni L. Validation of standard definition of sensitive versus refractory relapsed small cell lung cancer: a pooled analysis of topotecan second-line trials. Eur J Cancer. 2014;50 (13):2211-2218. doi:10.1016/j.ejca.2014.06.002

\section{Publish your work in this journal}

OncoTargets and Therapy is an international, peer-reviewed, open access journal focusing on the pathological basis of all cancers, potential targets for therapy and treatment protocols employed to improve the management of cancer patients. The journal also focuses on the impact of management programs and new therapeutic agents and protocols on patient perspectives such as quality of life, adherence and satisfaction. The manuscript management system is completely online and includes a very quick and fair peer-review system, which is all easy to use. Visit http://www.dovepress.com/ testimonials.php to read real quotes from published authors. 